\title{
18F-SMBT-1: A Selective and Reversible PET Tracer for Monoamine Oxidase-B Imaging
}

\author{
Ryuichi Harada ${ }^{1,2}$, Yoshimi Hayakawa ${ }^{3}$, Michinori Ezura ${ }^{4}$, Pradith Lerdsirisuk ${ }^{3}$, Yiqing Du ${ }^{1}$, Yoichi Ishikawa ${ }^{3}$, \\ Ren Iwata ${ }^{3}$, Miho Shidahara ${ }^{5}$, Aiko Ishiki ${ }^{2}$, Akio Kikuchi $^{4}$, Hiroyuki Arai ${ }^{2}$, Yukitsuka Kudo ${ }^{2}$, Kazuhiko Yanai $^{1,3}$, \\ Shozo Furumoto ${ }^{3}$, and Nobuyuki Okamura ${ }^{3,6}$ \\ ${ }^{I}$ Department of Pharmacology, Tohoku University School of Medicine, Sendai, Japan; ${ }^{2}$ Department of Geriatrics and Gerontology, \\ Division of Brain Sciences, Institute of Development, Aging, and Cancer, Tohoku University, Sendai, Japan; ${ }^{3}$ Cyclotron and \\ Radioisotope Center, Tohoku University, Sendai, Japan; ${ }^{4}$ Department of Neurology, Tohoku University Graduate School of Medicine. \\ 1-1 Seiryo-machi, Aoba-ku, Sendai, Japan; ${ }^{5}$ Department of Quantum Science and Energy Engineering, Tohoku University, Sendai, \\ Japan; and ${ }^{6}$ Division of Pharmacology, Faculty of Medicine, Tohoku Medical and Pharmaceutical University, Sendai, Japan
}

Reactive astrocytes play a key role in the pathogenesis of various neurodegenerative diseases. Monoamine oxidase- $\mathrm{B}$ (MAO-B) is one of the promising targets for the imaging of astrogliosis in the human brain. A novel selective and reversible MAO-B tracer, (S)-(2-methylpyrid5 -yl)-6-[(3-18 F-fluoro-2-hydroxy)propoxy]quinoline ( $\left.{ }^{18} \mathrm{~F}-\mathrm{SMBT}-1\right)$, was successfully developed via lead optimization from the first-generation tau PET tracer ${ }^{18} \mathrm{~F}-\mathrm{THK}-5351$. Methods: SMBT-1 was radiolabeled with ${ }^{18} \mathrm{~F}$ using the corresponding precursor. The binding affinity of radiolabeled compounds to MAO-B was assessed using saturation and competitive binding assays. The binding selectivity of ${ }^{18} \mathrm{~F}-\mathrm{SMBT}-$ 1 to MAO-B was evaluated by autoradiography of frozen human brain tissues. The pharmacokinetics and metabolism were assessed in normal mice after intravenous administration of ${ }^{18} \mathrm{~F}-\mathrm{SMBT}-1$. A $14-$ d toxicity study after the intravenous administration of ${ }^{18} \mathrm{~F}-\mathrm{SMBT}-1$ was performed using rats and mice. Results: In vitro binding assays demonstrated a high binding affinity of ${ }^{18} \mathrm{~F}-\mathrm{SMBT}-1$ to MAO-B (dissociation constant, $3.7 \mathrm{nM}$ ). In contrast, it showed low binding affinity to MAO-A and protein aggregates such as amyloid- $\beta$ and tau fibrils. Autoradiographic analysis showed higher amounts of ${ }^{18} \mathrm{~F}-\mathrm{SMBT}-1$ binding in the Alzheimer disease brain sections than in the control brain sections. ${ }^{18} \mathrm{~F}-\mathrm{SMBT}-1$ binding was completely displaced with the reversible MAO-B inhibitor lazabemide, demonstrating the high selectivity of ${ }^{18} \mathrm{~F}-\mathrm{SMBT}-1$ for MAO-B. Furthermore, ${ }^{18} \mathrm{~F}-\mathrm{SMBT}-1$ showed a high uptake by brain, rapid washout, and no radiolabeled metabolites in the brain of normal mice. ${ }^{18} \mathrm{~F}-\mathrm{SMBT}-1$ showed no significant binding to various receptors, ion channels, or transporters, and no toxic effects related to its administration were observed in mice and rats. Conclusion: ${ }^{18} \mathrm{~F}-\mathrm{SMBT}-1$ is a promising and selective MAO-B PET tracer candidate, which would be useful for quantitative monitoring of astrogliosis in the human brain.

Key Words: MAO-B; radiotracers; PET; molecular imaging

J Nucl Med 2021; 62:253-258

DOI: 10.2967/jnumed.120.244400

\footnotetext{
Received Mar. 12, 2020; revision accepted Jun. 15, 2020.

For correspondence or reprints contact: Ryuichi Harada, Department of Pharmacology, Tohoku University School of Medicine, 2-1, Seiryo-machi, Aoba-ku, Sendai 9808575 , Japan.

E-mail: ryuichi.harada.c8@med.tohoku.ac.jp

Published online Jul. 9, 2020.

COPYRIGHT @ 2021 by the Society of Nuclear Medicine and Molecular Imaging.
}

$\mathbf{R}$ eactive astrocytosis is the astrocyte response observed in various neurologic disorders. It is characterized by distinct morphologic alternations such as enlarged cell bodies and overexpression of glial fibrillary acidic protein, vimentin, and nestin (1). In neurodegenerative conditions, astrocytes turn reactive and secrete neurotoxic inflammatory cytokines (1). Reactive astrocytes also overexpress monoamine oxidase-B (MAO-B) in the outer membrane of the mitochondria. MAO-B, a major enzyme that metabolizes dopamine and histamine, is widely distributed in the human brain. Higher levels of MAO-B expression are physiologically observed in the basal forebrain, substantia nigra, basal ganglia, thalamus, and hippocampal uncus relative to the cerebellar cortex (2). MAO-B levels in whole brain regions also increase linearly in an age-dependent manner during normal aging processes $(2,3)$. Elevated MAO-B levels in autopsy-confirmed Alzheimer disease (AD) brains were observed during in vitro binding studies with selective MAO-B radioligands, such as ${ }^{3} \mathrm{H}$-L-deprenyl and ${ }^{3} \mathrm{H}$-lazabemide (4-6). In addition, MAO-B elevation was also observed in the postmortem brains of parkinsonian syndromes, progressive supranuclear palsy, and multiple-system atrophy (7). This finding indicates that MAO-B could be an attractive target for visualizing reactive astrocytes in vivo during neuroinflammatory processes.

Much effort has been made to develop novel MAO-B PET tracers based on the chemical structure of selective MAO-B inhibitors $(8,9) .{ }^{11} \mathrm{C}$-L-deprenyl- $\mathrm{D}_{2}$ is the most commonly used PET tracer for imaging MAO-B in the human brain (Fig. 1). However, quantifying ${ }^{11} \mathrm{C}$-L-deprenyl- $\mathrm{D}_{2}$ binding is difficult because of its irreversible binding. ${ }^{11} \mathrm{C}-\mathrm{SL} 25.1188$, a reversible MAO-B PET tracer, was developed and used in human studies (10). The short half-life of ${ }^{11} \mathrm{C}$ (20 min) limits the clinical utility of these PET tracers. Therefore, several ${ }^{18} \mathrm{~F}$-labeled PET tracers have been developed for imaging MAO-B (11-14).

${ }^{18} \mathrm{~F}-\mathrm{THK}-5351$ was originally designed to detect neurofibrillary tangles in vivo and was found to bind MAO-B with high affinity $(15,16)$. Clinical ${ }^{18} \mathrm{~F}-\mathrm{THK}-5351$ PET studies demonstrated high tracer retention in sites susceptible to astrogliosis in various neurodegenerative conditions (17-26). The nonselective binding of THK-5351 to MAO-B and tau limits its clinical utility as a biomarker. Lead optimization for generating a selective MAO-B binding tracer led to the development of $(S)$-(2-methylpyrid-5yl)-6-[(3- ${ }^{18}$ F-fluoro-2-hydroxy)propoxy]quinoline ( $\left.{ }^{18} \mathrm{~F}-\mathrm{SMBT}-1\right)$ (Fig. 1), 


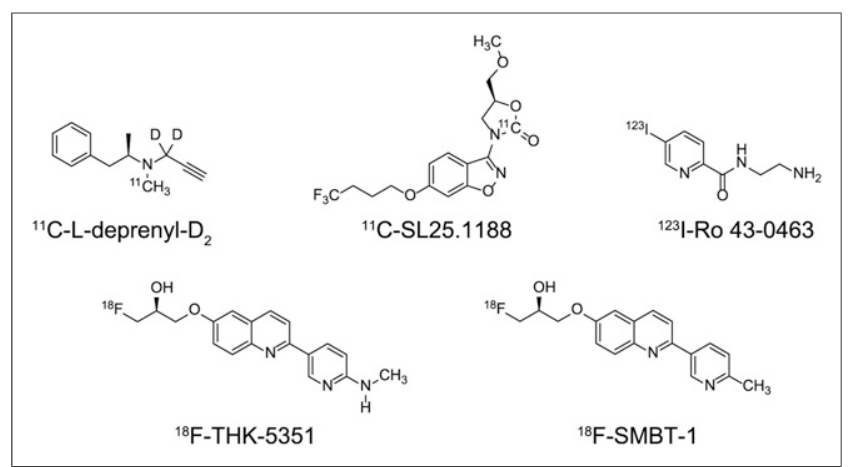

FIGURE 1. Chemical structures of MAO-B tracers tested in humans, ${ }^{18} \mathrm{~F}-\mathrm{THK}-5351$ and ${ }^{18} \mathrm{~F}-\mathrm{SMBT}-1$.

a novel radiolabeled tracer compound. Here, we report the preclinical binding, pharmacokinetics, and metabolic properties of ${ }^{18} \mathrm{~F}$ SMBT-1 and its derivatives.

\section{MATERIALS AND METHODS}

\section{Synthesis of ${ }^{18} \mathrm{~F}-\mathrm{SMBT}-1$ and Its Derivatives}

Methods for the synthesis and characterization of the tracer compounds are described in the supplemental data (Supplemental Schemes 1-4; supplemental materials are available at http://jnm.snmjournals.org).

\section{Radiochemistry}

${ }^{18} \mathrm{~F}$-fluoride, produced by the ${ }^{18} \mathrm{O}(\mathrm{p}, \mathrm{n}){ }^{18} \mathrm{~F}$ reaction on enriched ${ }^{18} \mathrm{O}$ $\mathrm{H}_{2} \mathrm{O}$ with a cyclotron, was separated from the irradiated target with a Sep-Pak Light Accell Plus QMA cartridge (Waters), which was washed in advance with $\mathrm{K}_{2} \mathrm{CO}_{3}$ followed by water. The trapped ${ }^{18} \mathrm{~F}$ fluoride was eluted with a solution of Kryptofix 222 (8 mg; Merck), $\mathrm{K}_{2} \mathrm{CO}_{3}(1.5 \mathrm{mg})$, acetonitrile $(0.45 \mathrm{~mL})$, and water $(0.13 \mathrm{~mL})$. The eluted solution was then used for subsequent radiofluorination. The solution was evaporated to dryness by azeotropic distillation with a helium flow $(300 \mathrm{~mL} / \mathrm{min})$, heating at $110^{\circ} \mathrm{C}$, and stirring. After drying, the tosylate precursor $(S)$-(2-methylpyrid-5-yl)-6-[[2-(tetrahydro2H-pyran-2-yloxy)-3-tosyloxy]propoxy]quinoline (THK-5475; $2 \mathrm{mg}$ ) dissolved in dimethylsulfoxide $(0.45 \mathrm{~mL})$ was transferred and stirred at $110^{\circ} \mathrm{C}$ for $10 \mathrm{~min}$. Next, hydrochloride aqueous solution $(2 \mathrm{M}, 0.2$ $\mathrm{mL}$ ) was added to the reaction solution and stirred at $110^{\circ} \mathrm{C}$ for an additional $3 \mathrm{~min}$ for deprotection of the hydroxy group. The reaction was then quenched with potassium acetate aqueous solution $(0.2 \mathrm{M}$, $4 \mathrm{~mL}$ ), followed by solid-phase extraction. The crude mixture was passed through the activated Sep-Pak tC18 Plus (Waters) and then washed with water. Radioactive products remaining in the solid phase were eluted with $70 \%$ ethanol $(0.7 \mathrm{~mL})$ and diluted with water $(0.12 \mathrm{~mL})$ and then subjected to semipreparative high-performance liquid chromatography (column, Inertsil ODS-4 (GL Sciences, Inc.; mobile phase, $20 \mathrm{mmol} / \mathrm{L} \mathrm{NaH} \mathrm{PO}_{4}$ /acetonitrile [67/33 for $\left.{ }^{18} \mathrm{~F}-\mathrm{SMBT}-1\right]$; flow rate, $5.0 \mathrm{~mL} / \mathrm{min}$ ). A detailed account of the optimized procedure is provided in Supplemental Table 1. ${ }^{18} \mathrm{~F}-\mathrm{SMBT}-1$ was also produced by 1 -pot microscale radiosynthesis (27). ${ }^{18} \mathrm{~F}-\mathrm{THK}-5351$ was prepared as described previously (15). ${ }^{18} \mathrm{~F}$-fluoroethyl harmine was prepared from the corresponding precursor using the microscale radiosynthesis method, as previously described (27). ${ }^{125}$ I-Ro 43-0463 was prepared as previously described (11). ${ }^{18} \mathrm{~F}$-SMBT-1, ${ }^{18} \mathrm{~F}$-THK-5351, ${ }^{18} \mathrm{~F}$-fluoroethyl harmine, and ${ }^{125} \mathrm{I}-\mathrm{Ro}$ 43-0463 were obtained in greater than $95 \%$ radiochemical purity after high-performance liquid chromatography purification. The averages of decay-corrected radiochemical yields and molar activity at the end of ${ }^{18} \mathrm{~F}$-SMBT-1 synthesis were $39 \%$ and $414 \mathrm{GBq} / \mu \mathrm{mol}$, respectively.

\section{In Vitro Binding Studies}

${ }^{3} \mathrm{H}$-THK-5351 (molar activity, $2.96 \mathrm{TBq} / \mathrm{mmol}$; radiochemical purity, 98.9\%) was custom-labeled by Sekisui Medical Inc. ${ }^{3} \mathrm{H}$-Pittsburgh compound B (molar activity, $2.96 \mathrm{TBq} / \mathrm{mmol}$; radiochemical purity, 99\%) was obtained from American Radiolabeled Chemicals. ${ }^{3} \mathrm{H}-\mathrm{MK}-$ 6240 (molar activity, $0.83 \mathrm{TBq} / \mathrm{mmol}$; radiochemical purity, 99\%) was obtained from ViTrax. Competitive binding assays were performed as previously described (28). ${ }^{3} \mathrm{H}$-Pittsburgh compound $\mathrm{B}(1 \mathrm{nM})$ and ${ }^{3} \mathrm{H}-$ MK-6240 (1.5 nM) were used as radioligands for amyloid and tau aggregates, respectively. An in vitro saturation binding assay was also performed using ${ }^{18} \mathrm{~F}$-labeled compounds as previously described (29). ${ }^{18}$ F-fluoroethyl harmine and recombinant MAO-A (M7316; SigmaAldrich) were used for an in vitro competitive binding assay against MAO-A. The off-rate was determined as previously described (30). All assays were performed at room temperature in quadruplicate, and the off-rate was determined by GraphPad Prism, version 7.0. Correlation analysis between tracer binding and MAO-B activity was performed as previously described (16).

\section{In Vitro Autoradiography}

The Ethics Committee of the Tohoku University Graduate School of Medicine approved this study, and all subjects provided written informed consent. Postmortem brain sections from control subjects and $\mathrm{AD}$ patients were acquired from Tohoku University Brain Bank. In vitro autoradiography was performed as previously described (16). To account for MAO-B binding in the brain tissues, the reaction was incubated in the presence of the MAO-B inhibitor lazabemide $(1 \mu \mathrm{M})$. After postfixation in $4 \%$ paraformaldehyde for $30 \mathrm{~min}$, adjacent frozen sections were immunostained with anti-MAO-B (1:400; Sigma-Aldrich), antitau (AT8; Innogenetics), and anti- $\beta$-amyloid (6F/3D; Dako) antibodies.

\section{Biodistribution Study in Normal Mice}

All animal experimental protocols were approved by the Laboratory Animal Care Committee of Tohoku University. The biodistribution study was performed as previously described (29). The radiation dose and mass dose for humans were estimated on the basis of the biodistribution data from mice (31).

\section{Metabolite Analysis}

Male Institute of Cancer Research (ICR) mice (6 wk old) were killed by decapitation under anesthesia at 2, 10, and $30 \mathrm{~min}$ after intravenous administration of ${ }^{18} \mathrm{~F}-\mathrm{SMBT}-1$ (19.4 MBq), and the brain and cardiac blood were collected. Extraction and metabolite analysis were performed as previously described (32). To identify the major metabolite of ${ }^{18} \mathrm{~F}$-SMBT-1, in vitro enzyme assays were performed using human sulfotransferase 1E1 (Cypex Ltd.) as described previously (28).

\section{Receptor Binding Assays}

Receptor binding screen assays were performed by Sekisui Medical Inc. Binding inhibition (\%) was determined by competitive radioligand assays against 60 common neurotransmitter receptors, ion channels, and transporters, as previously described (29).

\section{Animal Toxicity Studies}

Acute toxicity studies were performed on Sprague-Dawley rats and ICR mice. A single intravenous dose of ${ }^{18} \mathrm{~F}-\mathrm{SMBT}-1$ was administered by LSI Medience Inc., as previously described (29).

\section{RESULTS}

\section{In Vitro Competitive Binding Studies}

We performed an in vitro competitive binding assay using ${ }^{3} \mathrm{H}$ THK-5351 to measure the binding affinity of various compounds against recombinant MAO-B (Supplemental Table 2). Arylquinoline derivatives including THK-5105 showed high binding affinity 
to MAO-B. The binding affinity of THK-5105 to MAO-B was substantially reduced after substitution of the hydroxy group in the (3-fluoro-2-hydroxy)propoxyl group by hydrogen. The chirality of compounds was also associated with the binding affinity to MAO-B. For example, $R$-enantiomer (THK-5451) showed lower affinity to MAO-B than $S$-enantiomer (THK-5351). Previous analysis of the structure-activity relationship of 2-arylquinoline derivatives showed that the 2 amino groups on the pyridine ring were essential for binding to tau aggregates (Ryuichi Harada, unpublished data, November 2013). We therefore investigated the substituent on the pyridine ring at the 2-position to ensure high selectivity of compounds for MAO-B over tau aggregates. Although the hydrogen substituent on the pyridine ring (SMBT-0) reduced the binding affinity to MAO-B, the methyl substituent (SMBT-1) maintained a high binding affinity to MAO-B at a level comparable to THK-5351 and several other MAO-B inhibitors, such as rasagiline, lazabemide, and safinamide. Our SAR study of ${ }^{18} \mathrm{~F}-\mathrm{SMBT}-1$ derivatives demonstrated that the 2-methylpyridine derivative was the most ideal for generating high binding affinity to MAO-B (Supplemental Table 2). The binding of ${ }^{18} \mathrm{~F}-\mathrm{SMBT}-1$ to recombinant MAO-A was further investigated by an in vitro competitive binding assay using the reversible MAO-A binder ${ }^{18} \mathrm{~F}$-fluoroethyl harmine. In contrast with its high affinity to MAO-B, ${ }^{18} \mathrm{~F}-\mathrm{SMBT}-1$ showed low binding affinity to MAO-A (half-maximal inhibitory concentration, $713 \mathrm{nM}$ ). Furthermore, ${ }^{18} \mathrm{~F}-\mathrm{SMBT}-1$ showed low binding affinity to amyloid- $\beta$ and tau protein aggregates (half-maximal inhibitory concentration, $>1,000$ nM) (Supplemental Table 3). Receptor binding screen assays demonstrated no remarkable interaction with various receptors, ion channels, and transporters (Supplemental Table 4).

\section{In Vitro Binding Studies of ${ }^{18} \mathrm{~F}-\mathrm{SMBT}-1$ to MAO-B}

Next, we radiolabeled SMBT-1 with ${ }^{18} \mathrm{~F}$ and investigated the binding properties of ${ }^{18} \mathrm{~F}-\mathrm{SMBT}-1$ to MAO-B in detail. Saturation binding assays demonstrated a high binding affinity of ${ }^{18} \mathrm{~F}-\mathrm{SMBT}$ 1 for recombinant MAO-B (dissociation constant $\left[K_{\mathrm{D}}\right]=3.7 \mathrm{nM}$; number of binding sites $\left[B_{\max }\right]=110.4 \mathrm{pmol} / \mathrm{mg}$ protein) (Fig. 2). As shown in Supplemental Figure 1, ${ }^{18}$ F-SMBT-1 also showed high binding affinity for MAO-B-rich $\mathrm{AD}$ brain homogenates $\left(K_{\mathrm{D}}=3.5\right.$ $\mathrm{nM})$ and mouse brain homogenates $\left(K_{\mathrm{D}}=4.3 \mathrm{nM}\right)$. However, the $B_{\max }$ in $\mathrm{AD}$ brain homogenates ( $606 \mathrm{pmol} / \mathrm{g}$ of tissue) was approximately 3 times higher than that in mouse brain homogenates $(153 \mathrm{pmol} / \mathrm{g}$ of tissue). The specific binding of ${ }^{18} \mathrm{~F}-\mathrm{SMBT}-1$ to human $\mathrm{AD}$ brain homogenates and recombinant MAO-B was reversible with similar kinetics (Fig. 3B). The off-rates were 0.0087 and $0.0072 \mathrm{~min}^{-1}$ for human $\mathrm{AD}$ brain homogenate and recombinant $\mathrm{MAO}-\mathrm{B}$, respectively.
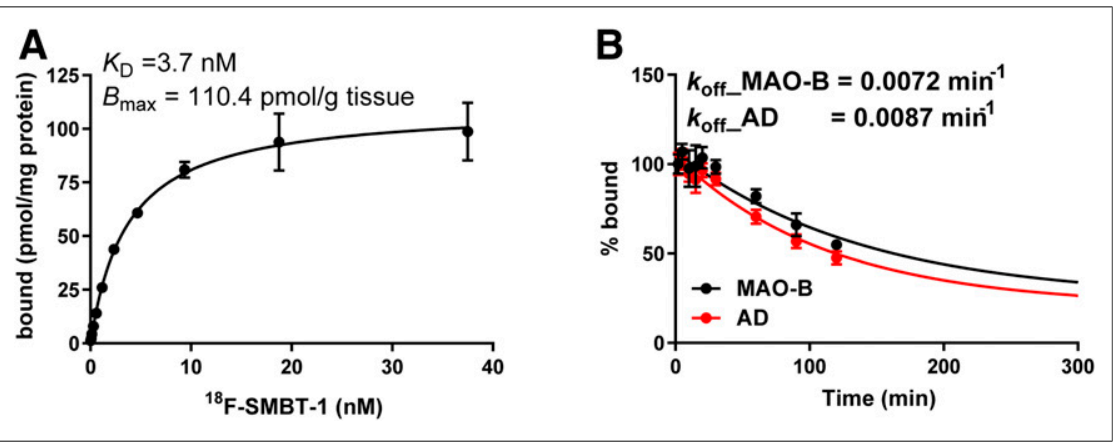

FIGURE 2. In vitro saturation binding of ${ }^{18} \mathrm{~F}-\mathrm{SMBT}-1$ against recombinant MAO-B (A). In vitro binding kinetics of ${ }^{18} \mathrm{~F}-\mathrm{SMBT}-1$ against recombinant MAO-B and $A D$ brain homogenate $(\mathrm{B})$.
A strong correlation was observed between regional in vitro ${ }^{18} \mathrm{~F}$ SMBT-1 binding and MAO-B activity in a patient with AD (Spearman $r=0.923, P<0.0001$; Fig. 3). We also observed a significant correlation with sarkosyl insoluble tau (Spearman $r=0.678, P=$ 0.0049); however, this association was weaker than that observed with MAO-B activity. In contrast, no significant correlation was observed with insoluble total A $\beta$ (Spearman $r=-0.391, P=0.135$ ).

\section{In Vitro Autoradiography of Postmortem Human Brain Sections}

In vitro autoradiography of ${ }^{18} \mathrm{~F}-\mathrm{SMBT}-1$ was conducted to further evaluate the binding selectivity of ${ }^{18} \mathrm{~F}-\mathrm{SMBT}-1$ in human brain sections. Specific binding of ${ }^{18} \mathrm{~F}-\mathrm{SMBT}-1$ was greater in human AD brain sections than in control brain sections, as was consistent with the results of MAO-B immunohistochemistry (Fig. 4). This specific binding of ${ }^{18} \mathrm{~F}-\mathrm{SMBT}-1$ was completely displaced in the presence of the selective MAO-B inhibitor lazabemide, although ${ }^{18} \mathrm{~F}-\mathrm{THK}-5351$ binding remained detectable in human AD brain sections after lazabemide treatment. The distribution of ${ }^{18} \mathrm{~F}-\mathrm{SMBT}-1$ was also consistent with that of the reversible MAO-B tracer, ${ }^{125}$ I-Ro 43-0463 (Supplemental Fig. 2). Considerable amounts of ${ }^{3} \mathrm{H}$-Pittsburgh compound B and ${ }^{3} \mathrm{H}-\mathrm{MK}-6240$ binding were detected in these sections. These results indicate that ${ }^{18} \mathrm{~F}-\mathrm{SMBT}-1$ binds to MAO-B with high selectivity.

\section{Biodistribution of ${ }^{18} \mathrm{~F}-\mathrm{SMBT}-1$ in Mice}

${ }^{18} \mathrm{~F}-\mathrm{SMBT}-1$ showed excellent blood-brain barrier permeability in mice. The peak uptake of ${ }^{18} \mathrm{~F}-\mathrm{SMBT}-1(7.85 \% \pm 0.76 \%$ injected dose/g at 2 min after injection) by the brain was greater than that of ${ }^{18} \mathrm{~F}-\mathrm{THK}-5351$. Furthermore, ${ }^{18} \mathrm{~F}-\mathrm{SMBT}-1$ showed rapid washout from normal brain tissue and no significant defluorination in mice (Table 1). ${ }^{18} \mathrm{~F}-\mathrm{SMBT}-1$ radiation exposure was estimated from the biodistribution data from mice (Supplemental Table 5). The resultant whole-body effective dose equivalents were 12.2 $\mu \mathrm{Sv} / \mathrm{MBq}$ (male) and $21.3 \mu \mathrm{Sv} / \mathrm{MBq}$ (female), which were comparable to those of ${ }^{18} \mathrm{~F}-\mathrm{THK}-5351$ (15).

\section{In Vivo Metabolism in Mice}

The extraction efficiencies from blood and brain were $89 \%$ and $87 \%$, respectively. Figure 5 shows the time-activity kinetics of radiolabeled metabolites and ${ }^{18} \mathrm{~F}-\mathrm{SMBT}-1$ in the brain and plasma. ${ }^{18} \mathrm{~F}-\mathrm{SMBT}-1$ was dominantly metabolized to 1 polar metabolite. In plasma, $92 \%, 65 \%$, and $19 \%$ of the parent compound remained at 2, 10, and $30 \mathrm{~min}$ after injection, respectively. On the other hand, most of the radioactivity in the brain was derived from the parent compound during the 30-min period after intravenous administration. As previously observed in ${ }^{18} \mathrm{~F}$-THK5351 (28), the major metabolite M2 exhibited the same $R_{\mathrm{f}}$ value as enzymatically produced $O$-sulfated ${ }^{18} \mathrm{~F}$-SMBT-1.

\section{Animal Toxicity Studies}

A single intravenous administration of ${ }^{18} \mathrm{~F}-$ SMBT-1 at $1 \mathrm{mg} / \mathrm{kg}$, equivalent to $100,000-$ fold the intended clinical dose for humans, caused no systemic toxicity in rats or mice.

\section{DISCUSSION}

${ }^{18} \mathrm{~F}-\mathrm{SMBT}-1$ is a single $S$-enantiomer and 2-methylpyridine derivative of ${ }^{18} \mathrm{~F}$-THK-5351. ${ }^{18} \mathrm{~F}-\mathrm{THK}-5351$ was originally designed to detect tau aggregates in the form of paired 


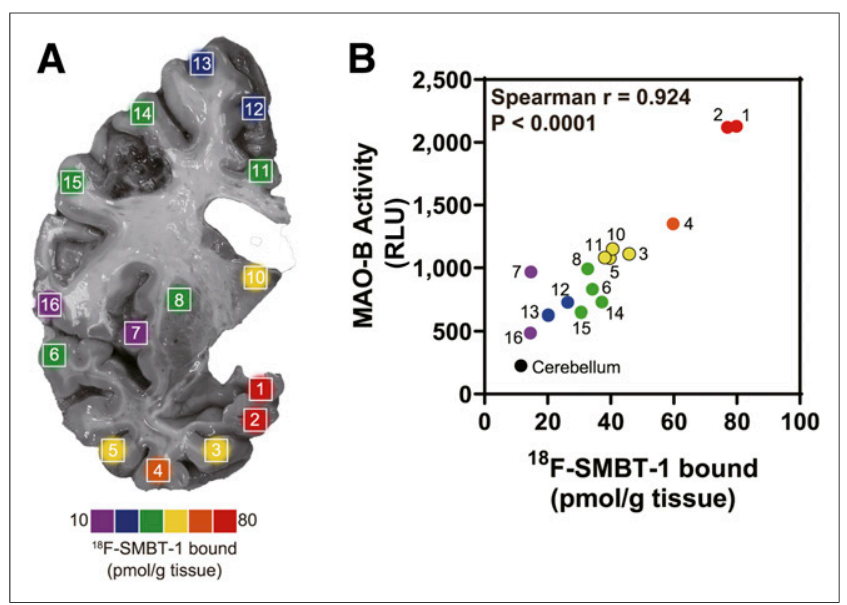

FIGURE 3. ${ }^{18} \mathrm{~F}-\mathrm{SMBT}-1$ binding density map in 16 regions of coronal brain section of autopsy tissue from right hemisphere from patient with $A D$ (81-y-old man) (A). Correlation of regional in vitro ${ }^{18} \mathrm{~F}-\mathrm{SMBT}-1$ binding and MAO-B activity (B). RLU = relative luminescence units.

helical filaments. However, recent studies showed high amounts of ${ }^{18}$ F-THK-5351 binding to MAO-B. Recent clinical studies have demonstrated the unique pattern of ${ }^{18} \mathrm{~F}-\mathrm{THK}-5351$ binding in various neurodegenerative diseases other than AD. However, the lack of binding selectivity of this tracer makes it difficult to interpret PET images. Therefore, we conducted lead optimization testing on THK-5351 derivatives to determine a selective MAO-B binding profile and a favorable pharmacokinetics profile. Previous structure-activity relationship analysis of 2-arylquinoline derivatives showed that the 2-amino group on the pyridine ring of 2-arylquinoline derivatives was essential for binding to tau aggregates. In the present study, structure-activity relationship analysis of MAO-B revealed that the hydroxy group in the (3-fluoro-2-hydroxy)propoxyl group of 2-arylquinoline plays an important role in achieving high binding affinity for MAO-B; (3-fluoro-2-hydroxy)propoxyl group has a chiral center. Previous studies demonstrated that the $S$-enantiomers of THK compounds showed better pharmacokinetics than the $R$ enantiomers $(30,32)$. In addition, the present study demonstrated that the affinity of the $S$-enantiomer for MAO-B was higher than that of the $R$-enantiomer. Therefore, we selected the $S$-enantiomer as the novel MAO-B tracer candidate. We further explored the functional group at position 2 on the pyridine ring to reduce the binding affinity for tau aggregates. Among several derivatives, ${ }^{18} \mathrm{~F}$ SMBT-1 showed the highest affinity for MAO-B and an excellent binding selectivity for MAO-B over MAO-A, amyloid, and tau aggregates. These findings were supported by in vitro autoradiography of ${ }^{18} \mathrm{~F}-\mathrm{SMBT}-1$ using human brain tissues.

The binding of the radiotracer can be predicted by the ratio of the $B_{\max }$ to the binding affinity $\left(K_{\mathrm{D}}\right)$ for the target. ${ }^{18} \mathrm{~F}-\mathrm{SMBT}-1$ showed low $K_{\mathrm{D}}$ and high $B_{\max }$ values $\left(K_{\mathrm{D}}=3.7 \mathrm{nM}, B_{\max }=110.4\right.$ $\mathrm{pmol} / \mathrm{mg}$ protein) against recombinant MAO-B and human brain homogenates $\left(K_{\mathrm{D}}=3.5 \mathrm{nM}, B_{\max }=606 \mathrm{pmol} / \mathrm{g}\right.$ tissue $)$. The binding potential $\left(B_{\max } / K_{\mathrm{D}}\right)$ was greater than that of currently available tau PET tracers, suggesting the ability of ${ }^{18} \mathrm{~F}-\mathrm{SMBT}-1$ to detect MAO-B in vivo. MAO-B levels in the human brain cortex were 2.5- to 4.7-fold higher than those in rodents (33). This finding was consistent with the $B_{\max }$ determined via in vitro ${ }^{18} \mathrm{~F}$ SMBT-1 binding assays. Since MAO-B is expressed predominantly in humans and not in rodents, the differences in MAO expression between these species should be considered when using ${ }^{18} \mathrm{~F}-\mathrm{SMBT}-1$ in preclinical studies.

Classic MAO-B PET tracers based on MAO-B inhibitors such as ${ }^{11} \mathrm{C}$-pargyline, ${ }^{11} \mathrm{C}$-L-deprenyl, and ${ }^{18} \mathrm{~F}$-rasagiline show irreversible binding to MAO-B. This irreversibility is due to the formation of a covalent adduct with flavin adenine dinucleotide, which is a redox-active coenzyme associated with MAO$\mathrm{B}$ (34-36). The irreversibility of tracer binding led to an underestimation of MAO-B in the high-concentration regions, as the rate of tracer binding may exceed the rate of tracer delivery (37). Deuterium-substituted radiotracers such as ${ }^{11} \mathrm{C}$-L-deprenyl- $\mathrm{D}_{2},{ }^{18} \mathrm{~F}$-fluorodeprenyl- $\mathrm{D}_{2}$, and ${ }^{18} \mathrm{~F}$-rasagiline- $\mathrm{D}_{2}$ reduce the trapping rate of the tracers and partly overcome these limitations; however, they still possessed irreversible binding properties for MAO-B. Reversible radiotracers would be preferable for sensitive and quantitative MAO-B detection in vivo. ${ }^{11} \mathrm{C}$ limits the use of the radiotracer to centers with an on-site cyclotron and ${ }^{11} \mathrm{C}$ radiochemistry expertise. ${ }^{18} \mathrm{~F}$-labeling of SMBT-1 permits centralized production and regional distribution of the radiotracer. The in vitro binding kinetics of ${ }^{18} \mathrm{~F}-\mathrm{SMBT}-1$ demonstrated its reversible binding to MAO-B in human brains. The advantages of ${ }^{18} \mathrm{~F}-\mathrm{SMBT}-1$ in terms of its reversibility would allow us to perform repeated PET scans on the same subject. This ability will help in longitudinal quantification of astrogliosis and measurement of MAO-B occupancy via therapeutics. 
TABLE 1

Biodistribution After Intravenous Injection of ${ }^{18} \mathrm{~F}-\mathrm{SMBT}-1$ in ICR Mice

\begin{tabular}{lccccc}
\hline \multicolumn{1}{c}{ Organ } & $2 \mathrm{~min}$ & $10 \mathrm{~min}$ & $30 \mathrm{~min}$ & $60 \mathrm{~min}$ & $120 \mathrm{~min}$ \\
\hline Blood & $3.20 \pm 0.45$ & $1.28 \pm 0.12$ & $0.52 \pm 0.10$ & $0.28 \pm 0.03$ & $0.17 \pm 0.04$ \\
Brain & $7.85 \pm 0.76$ & $2.11 \pm 0.35$ & $0.32 \pm 0.05$ & $0.20 \pm 0.02$ & $0.14 \pm 0.02$ \\
Liver & $7.97 \pm 1.72$ & $13.7 \pm 2.81$ & $3.16 \pm 1.10$ & $1.35 \pm 0.06$ & $1.67 \pm 1.27$ \\
Kidney & $6.77 \pm 1.86$ & $2.24 \pm 0.44$ & $0.73 \pm 0.21$ & $0.29 \pm 0.06$ & $0.21 \pm 0.07$ \\
Heart & $6.14 \pm 1.07$ & $1.61 \pm 0.17$ & $0.41 \pm 0.10$ & $0.21 \pm 0.05$ & $0.14 \pm 0.01$ \\
Lung & $6.56 \pm 1.96$ & $2.10 \pm 0.29$ & $0.45 \pm 0.14$ & $0.22 \pm 0.05$ & $0.14 \pm 0.04$ \\
Spleen & $2.03 \pm 0.34$ & $1.04 \pm 0.10$ & $0.27 \pm 0.05$ & $0.16 \pm 0.01$ & $0.12 \pm 0.05$ \\
Stomach & $3.91 \pm 0.82$ & $5.99 \pm 2.42$ & $5.32 \pm 0.92$ & $2.48 \pm 0.54$ & $0.98 \pm 0.23$ \\
Small intestine & $5.93 \pm 0.87$ & $21.1 \pm 5.28$ & $44.3 \pm 6.65$ & $56.5 \pm 17.5$ & $27.1 \pm 15.4$ \\
Large intestine & $2.14 \pm 0.21$ & $2.13 \pm 0.15$ & $1.92 \pm 0.24$ & $9.48 \pm 12.3$ & $50.4 \pm 14.0$ \\
Bladder & $1.46 \pm 0.53$ & $1.52 \pm 0.71$ & $2.17 \pm 2.24$ & $2.15 \pm 1.76$ & $1.34 \pm 0.81$ \\
Bone & $1.18 \pm 0.10$ & $0.73 \pm 0.10$ & $0.40 \pm 0.04$ & $0.66 \pm 0.16$ & $1.08 \pm 0.06$ \\
Muscle & $2.40 \pm 0.60$ & $1.10 \pm 0.13$ & $0.33 \pm 0.12$ & $0.23 \pm 0.06$ & $5.90 \pm 0.09$ \\
\hline Data are average $( \pm S D)$ percentage dose/g, in 4 mice. & & & \\
\hline
\end{tabular}

${ }^{18}$ F-SMBT-1 showed excellent pharmacokinetics profiles, such as high uptake by brain after intravenous administration and rapid washout from normal brain tissues without significant defluorination in mice. These characteristics were comparable to those of ${ }^{18} \mathrm{~F}-\mathrm{THK}-5351$. One of the drawbacks of ${ }^{11} \mathrm{C}$-L-deprenyl$\mathrm{D}_{2}$ is the existence of radiolabeled metabolites, such as $R(-)$ methamphetamine and $R(-)$-amphetamine, that can penetrate the blood-brain barrier and bind to monoamine transporters. Conversely, the reversible MAO-B PET tracer ${ }^{11} \mathrm{C}$-SL.25.1188 showed no radiolabeled metabolites capable of permeating the bloodbrain barrier. However, this tracer showed slow pharmacokinetics due to slow metabolism $(10,38)$. ${ }^{18} \mathrm{~F}$-SMBT-1 was completely metabolized in mice without leaving harmful radiolabeled metabolite remnants. The dominant radiolabeled metabolite (M2) in mouse plasma was $O$-sulfated ${ }^{18} \mathrm{~F}$-SMBT-1, which was not detectable in the mouse brain. A previous study demonstrated

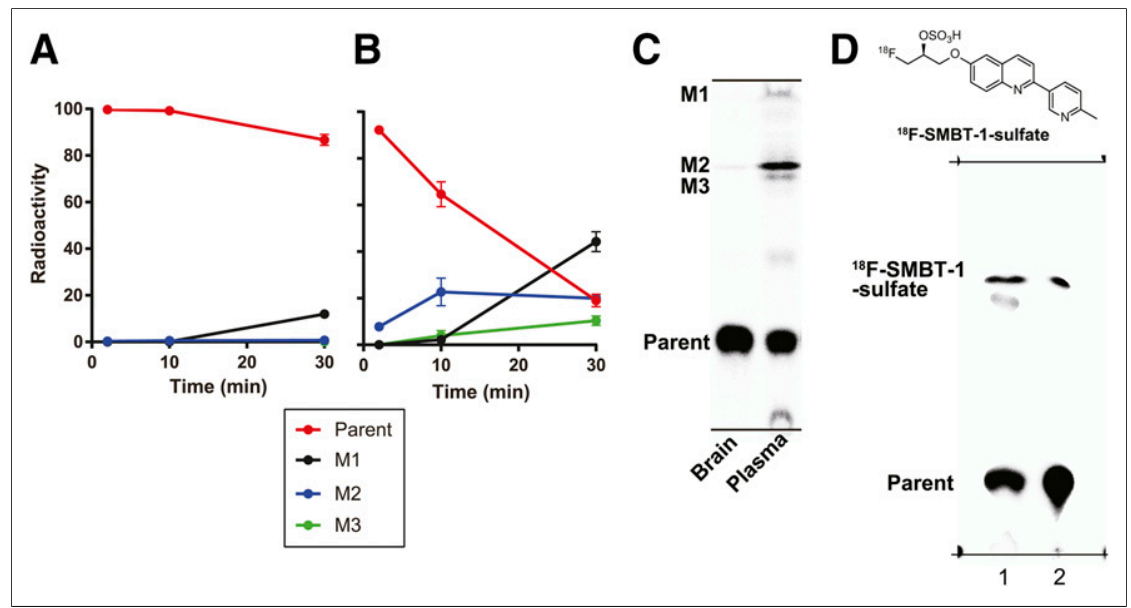

FIGURE 5. (A and B) Time-activity curves of parent and radiolabeled metabolites in brain (A) and plasma (B). (C) Reverse-phase thin-layer chromatograms. (D) Chemical structure of $\mathrm{O}$-sulfated ${ }^{18} \mathrm{~F}$ SMBT-1 by human sulfotransferase $1 \mathrm{E} 1$ and reverse-phase thin-layer chromatograms. Lane 1: mouse plasma at 10 min after injection of ${ }^{18} \mathrm{~F}-\mathrm{SMBT}-1$; lane $2:{ }^{18} \mathrm{~F}-\mathrm{SMBT}-1$ with human sulfotransferase $1 \mathrm{E} 1$. that $O$-sulfated THK-5351 did not penetrate the blood-brain barrier in mice and showed little binding to human brain homogenates (28). This evidence suggests that the in vivo metabolic profile of ${ }^{18} \mathrm{~F}$ SMBT-1 could be suitable for MAO-B imaging in the brain.

\section{CONCLUSION}

${ }^{18} \mathrm{~F}-\mathrm{SMBT}-1$ is a promising candidate as a selective and reversible imaging tracer for MAO-B. Ongoing clinical study will allow us to classify the clinical utility of this tracer in vivo.

\section{DISCLOSURE}

This study was supported by Cino Ltd., a Grant-in-Aid for Young Scientists (18K15538), a Grant-in-Aid for Scientific Research (B) (18H02771), a Grant-in-Aid for Scientific Research on Innovative Areas (Brain Protein Aging and Dementia Control) (26117003), a Grant-in-Aid for Fostering Joint International Research (B) (19KK0212) from MEXT, the Strategic Research Program for Brain Science (JP19dm0107157) from the Japan Agency for Medical Research and Development, and a Grant-in-Aid for Joint Research by Young Researchers from Tohoku University Graduate School of Medicine and Shimadzu Science Foundation. Yukitsuka Kudo and Nobuyuki Okamura own stock in Clino Ltd. Ryuichi Harada, Shozo Furumoto, Yukitsuka Kudo, and Nobuyuki Okamura have a patent pending for the technology described in this article. No other potential conflict of interest relevant to this article was reported.

\section{ACKNOWLEDGMENTS}

We thank the staff at the Cyclotron and Radioisotope Center of Tohoku University for the HM-12 cyclotron operation. 
We acknowledge the support of the Biomedical Research Core of Tohoku University Graduate School of Medicine.

KEY POINTS

QUESTION: How do the preclinical properties of a newly generated MAO-B tracer, ${ }^{18} \mathrm{~F}-\mathrm{SMBT}-1$, compare with those of ${ }^{18} \mathrm{~F}-\mathrm{THK}-$ 5351 ?

PERTINENT FINDINGS: $18 \mathrm{~F}-\mathrm{SMBT}-1$ possess high affinity and high selectivity for MAO-B in a reversible binding fashion, with preferable pharmacokinetic and metabolic profiles.

IMPLICATIONS FOR PATIENT CARE: ${ }^{18} \mathrm{~F}-\mathrm{SMBT}-1$ has potential for detecting $\mathrm{MAO}-\mathrm{B}-$ expressing astrocytosis in humans.

\section{REFERENCES}

1. Carter SF, Herholz K, Rosa-Neto P, Pellerin L, Nordberg A, Zimmer ER. Astrocyte biomarkers in Alzheimer's disease. Trends Mol Med. 2019;25:77-95.

2. Tong J, Meyer JH, Furukawa Y, et al. Distribution of monoamine oxidase proteins in human brain: implications for brain imaging studies. J Cereb Blood Flow Metab. 2013;33:863-871.

3. Fowler JS, Volkow ND, Wang GJ, et al. Age-related increases in brain monoamine oxidase B in living healthy human subjects. Neurobiol Aging. 1997;18: 431-435.

4. Jossan SS, Gillberg PG, d'Argy R, et al. Quantitative localization of human brain monoamine oxidase B by large section autoradiography using $\mathrm{L}-\left[{ }^{3} \mathrm{H}\right]$ deprenyl. Brain Res. 1991;547:69-76.

5. Saura J, Luque JM, Cesura AM, et al. Increased monoamine oxidase B activity in plaque-associated astrocytes of Alzheimer brains revealed by quantitative enzyme radioautography. Neuroscience. 1994;62:15-30.

6. Gulyás B, Pavlova E, Kasa P, et al. Activated MAO-B in the brain of Alzheimer patients, demonstrated by $\left[{ }^{11} \mathrm{C}\right]-\mathrm{L}$-deprenyl using whole hemisphere autoradiography. Neurochem Int. 2011;58:60-68.

7. Tong J, Rathitharan G, Meyer JH, et al. Brain monoamine oxidase B and A in human parkinsonian dopamine deficiency disorders. Brain. 2017;140: 2460-2474.

8. Fowler JS, Logan J, Shumay E, Alia-Klein N, Wang GJ, Volkow ND. Monoamine oxidase: radiotracer chemistry and human studies. J Labelled Comp Radiopharm. 2015;58:51-64.

9. Narayanaswami V, Drake LR, Brooks AF, et al. Classics in neuroimaging: development of PET tracers for imaging monoamine oxidases. ACS Chem Neurosci. 2019;10:1867-1871.

10. Rusjan PM, Wilson AA, Miler L, et al. Kinetic modeling of the monoamine oxidase $\mathrm{B}$ radioligand $\left[{ }^{11} \mathrm{C}\right] \mathrm{SL} 25.1188$ in human brain with high-resolution positron emission tomography. J Cereb Blood Flow Metab. 2014;34:883-889.

11. Bläuenstein P, Remy N, Buck A, et al. In vivo properties of N-(2-aminoethyl)-5halogeno-2-pyridinecarboxamide ${ }^{18} \mathrm{~F}$ - and ${ }^{123} \mathrm{I}$-labelled reversible inhibitors of monoamine oxidase B. Nucl Med Biol. 1998;25:47-52.

12. Nag S, Lehmann L, Kettschau G, et al. Development of a novel fluorine-18 labeled deuterated fluororasagiline ([F-18]fluororasagiline-D-2) radioligand for PET studies of monoamino oxidase B. Bioorg Med Chem. 2013;21:6634-6641.

13. Nag S, Fazio P, Lehmann L, et al. In vivo and in vitro characterization of a novel mao-b inhibitor radioligand, 18F-labeled deuterated fluorodeprenyl. J Nucl Med. 2016;57:315-320.

14. Dahl K, Bernard-Gauthier V, Nag S, et al. Synthesis and preclinical evaluation of $\left[{ }^{18} \mathrm{~F}\right] \mathrm{FSL} 25.1188$, a reversible PET radioligand for monoamine oxidase-B. Bioorg Med Chem Lett. 2019;29:1624-1627.

15. Harada R, Okamura N, Furumoto $S$, et al. ${ }^{18}$ F-THK5351: a novel PET radiotracer for imaging neurofibrillary pathology in Alzheimer disease. J Nucl Med. 2016;57: 208-214.
16. Harada R, Ishiki A, Kai H, et al. Correlations of ${ }^{18} \mathrm{~F}-\mathrm{THK} 5351$ PET with postmortem burden of tau and astrogliosis in Alzheimer disease. J Nucl Med. 2018; 59:671-674.

17. Ishiki A, Harada R, Okamura $\mathrm{N}$, et al. Tau imaging with $\left[{ }^{18} \mathrm{~F}\right] \mathrm{THK}-5351$ in progressive supranuclear palsy. Eur J Neurol. 2017;24:130-136.

18. Kikuchi A, Okamura N, Hasegawa T, et al. In vivo visualization of tau deposits in corticobasal syndrome by ${ }^{18}$ F-THK5351 PET. Neurology. 2016;87:2309-2316.

19. Kobayashi R, Hayashi H, Kawakatsu S, et al. $\left[{ }^{18} \mathrm{~F}\right] \mathrm{THK}-5351$ PET imaging in early-stage semantic variant primary progressive aphasia: a report of two cases and a literature review. BMC Neurol. 2018;18:109.

20. Lee H, Seo S, Lee SY, et al. $\left[{ }^{18} \mathrm{~F}\right]$-THK5351 PET imaging in patients with semantic variant primary progressive aphasia. Alzheimer Dis Assoc Disord. 2018; 32:62-69.

21. Ishibashi K, Miura Y, Hirata K, Toyohara J, Ishii K. ${ }^{18} \mathrm{~F}-\mathrm{THK} 5351$ PET can identify astrogliosis in multiple sclerosis plaques. Clin Nucl Med. 2020;45:e98-e100.

22. Schönecker S, Brendel M, Palleis C, et al. PET imaging of astrogliosis and tau facilitates diagnosis of parkinsonian syndromes. Front Aging Neurosci. 2019;11:249.

23. Tago T, Toyohara J, Sengoku R, Murayama S, Ishii K. Monoamine oxidase B binding of ${ }^{18} \mathrm{~F}$-thk5351 to visualize glioblastoma and associated gliosis: an autopsy-confirmed case. Clin Nucl Med. 2019;44:507-509.

24. Ishibashi K, Kameyama M, Tago T, Toyohara J, Ishii K. Potential use of ${ }^{18} \mathrm{~F}$ THK5351 PET to identify Wallerian degeneration of the pyramidal tract caused by cerebral infarction. Clin Nucl Med. 2017;42:e523-e524.

25. Kim HJ, Cho H, Park S, et al. THK5351 and flortaucipir PET with pathological correlation in a Creutzfeldt-Jakob disease patient: a case report. BMC Neurol. 2019; 19:211.

26. Huang KL, Hsu JL, Lin KJ, et al. Visualization of ischemic stroke-related changes on ${ }^{18} \mathrm{~F}-\mathrm{THK}-5351$ positron emission tomography. EJNMMI Res. 2018;8:62.

27. Iwata R, Pascali C, Terasaki K, Ishikawa Y, Furumoto S, Yanai K. Practical microscale one-pot radiosynthesis of ${ }^{18} \mathrm{~F}$-labeled probes. J Labelled Comp Radiopharm. 2018;61:540-549.

28. Harada R, Furumoto S, Tago T, et al. Characterization of the radiolabeled metabolite of tau PET tracer ${ }^{18}$ F-THK5351. Eur J Nucl Med Mol Imaging. 2016;43: 2211-2218.

29. Okamura N, Furumoto S, Harada R, et al. Novel ${ }^{18}$ F-labeled arylquinoline derivatives for noninvasive imaging of tau pathology in Alzheimer disease. $J$ Nucl Med. 2013;54:1420-1427.

30. Tago T, Furumoto S, Okamura N, et al. Preclinical evaluation of $\left[{ }^{18} \mathrm{~F}\right] \mathrm{THK}-5105$ enantiomers: effects of chirality on its effectiveness as a tau imaging radiotracer. Mol Imaging Biol. 2016;18:258-266.

31. Shidahara M, Tashiro M, Okamura N, et al. Evaluation of the biodistribution and radiation dosimetry of the ${ }^{18} \mathrm{~F}$-labelled amyloid imaging probe $\left[{ }^{18} \mathrm{~F}\right] \mathrm{FACT}$. EJNMMI Res. 2013;3:32.

32. Tago T, Furumoto S, Okamura N, et al. Structure-activity relationship of 2arylquinolines as PET imaging tracers for tau pathology in Alzheimer disease. J Nucl Med. 2016;57:608-614.

33. Saura J, Kettler R, Da Prada M, Richards JG. Quantitative enzyme radioautography with ${ }^{3} \mathrm{H}-\mathrm{Ro}$ 41-1049 and ${ }^{3} \mathrm{H}$-Ro $19-6327$ in vitro: localization and abundance of MAO-A and MAO-B in rat CNS, peripheral organs, and human brain. J Neurosci. 1992;12:1977-1999.

34. Ishiwata K, Ido T, Yanai K, et al. Biodistribution of a positron-emitting suicide inactivator of monoamine oxidase, carbon-11 pargyline, in mice and a rabbit. $J$ Nucl Med. 1985;26:630-636.

35. Fowler JS, MacGregor RR, Wolf AP, et al. Mapping human brain monoamine oxidase A and B with ${ }^{11} \mathrm{C}$-labeled suicide inactivators and PET. Science. 1987;235: $481-485$.

36. Nag S, Lehmann L, Kettschau G, et al. Synthesis and evaluation of $\left[{ }^{18}\right.$ F $]$ fluororasagiline, a novel positron emission tomography (PET) radioligand for monoamine oxidase B (MAO-B). Bioorg Med Chem. 2012;20:3065-3071.

37. Fowler JS, Wang GJ, Logan J, et al. Selective reduction of radiotracer trapping by deuterium substitution: comparison of carbon-11-L-deprenyl and carbon-11deprenyl-D2 for MAO B mapping. J Nucl Med. 1995;36:1255-1262.

38. Saba W, Valette H, Peyronneau MA, et al. $\left[{ }^{11} \mathrm{C}\right] \mathrm{SL} 25.1188$, a new reversible radioligand to study the monoamine oxidase type B with PET: preclinical characterisation in nonhuman primate. Synapse. 2010;64:61-69. 